\title{
Editorial
}

\section{Skull base surgery}

Frederick A. Boop, M.D.

Department of Neurosurgery, Semmes-Murphey Neurologic and Spine Institute, Memphis, Tennessee

In their paper in this issue, Hayhurst et al. ${ }^{1}$ report a single-institutional retrospective review over a 10-year period of 23 children with tumors of varying types who required skull base surgical approaches. The children ranged from 1 to 15 years of age. Fifty-seven percent of the children had benign histology, and half underwent complete resection of their tumors.

At a mean follow-up of 60 months, the authors reported 1 perioperative death and 2 children who died of disease progression. Progression-free survival was $95 \%$ at 1 year and $68 \%$ at 5 years. Fifty-nine percent of patients required adjuvant therapy. Interestingly, 1 child underwent subtotal resection of a clival chordoma, but did not receive adjuvant therapy and has not shown tumor progression during follow-up.

Two children suffered the complication of postoperative CSF leakage followed by meningitis, postmeningitic hydrocephalus, and shunt placement, and are cognitively impaired as a result. This result emphasizes the fact that CSF leakage, the most common complication of skull base surgery in children, can have serious long-term consequences. Alternatively, the authors report that $85 \%$ of the children remain in mainstream schooling without the need for special educational assistance.

This review, as have others, reminds us that recent technical advances - such as image guidance, better neurophysiological monitoring, endoscope-assisted minimally invasive surgery, and intraoperative imaging-can further reduce morbidity from these operations. The development of dedicated pediatric skull base surgical teams further improves the cosmetic and functional outcomes of these children by avoiding injury to rudimentary teeth, minimizing injury to the developing facial skeleton, and limiting radiation doses to growing basicranial structures. The last 3 columns of Table 2 demonstrate the relative insensitivity of the Late Effects Severity Score (LESS) in rating the functional outcomes of these children.

(http://thejns.org/doi/abs/10.3171/2012.10.PEDS12479)

\section{Disclosure}

The author reports no conflict of interest.

\section{Reference}

1. Hayhurst C, Williams D, Yousaf J, Richardson D, Pizer B, Mallucci C: Skull base surgery for tumors in children: longterm clinical and functional outcome. Clinical article. J Neurosurg Pediatr [epub ahead of print February 22, 2013. DOI: 10.3171/2013.1.PEDS12120]

\section{Response}

\author{
Caroline Hayhurst, F.R.C.S., ${ }^{1}$ \\ and Conor L. Mallucci, F.R.C.S. ${ }^{2}$
}

${ }^{1}$ Department of Neurosurgery, University Hospital of Wales, Cardiff; and ${ }^{2}$ Department of Neurosurgery, Alder Hey Children's NHS Foundation Trust, Liverpool, United Kingdom

We would like to thank Dr. Boop for his valuable comments on our paper. In particular, we welcome his support for the development of dedicated pediatric skull base multidisciplinary teams to reduce the morbidity associated with these procedures. We would also emphasize that given the rarity of skull base pathology in the pediatric population, the inclusion of an adult skull base neurosurgeon with a dedicated pediatric interest is an invaluable addition to the team and is the model used in our center.

We chose the LESS $^{1}$ as a measurement of functional outcome because it provides a reproducible measure of multiple health domains including neurological deficit, visual and auditory function, and endocrine dysfunction, all of which are highly pertinent after skull base surgery. The LESS in our series correlates well with the better-known Lansky Play-Performance scale score and educational performance. However, future studies using prospective neuropsychological and quality of life measures before and after skull base surgery in children will be important work.

\section{Reference}

1. Benesch M, Spiegl K, Winter A, Passini A, Lackner H, Moser $\mathrm{A}$, et al: A scoring system to quantify late effects in children after treatment for medulloblastoma/ependymoma and its correlation with quality of life and neurocognitive functioning. Childs Nerv Syst 25:173-181, 2009

Please include this information when citing this paper: published online February 22, 2013; DOI: 10.3171/2012.10.PEDS12479. 